\title{
Linear closed-loop control using Luenberger observer applied to insulin administration for type 1 diabetes
}

\author{
Onofre Orozco-López ${ }^{1}$, Carlos E. Castañeda ${ }^{1}$, Agustín Rodrí1guez-Herrero ${ }^{2,3}$, Gema \\ García-Saéz $^{2,3}$, María Elena Hernando ${ }^{2,3}$
}

\begin{abstract}
In this work the design of a Luenberger observer is proposed to estimate the unmeasurable state space variables from Hovork's model. This model is linearized and evaluated in an operation point where Luenberger observer is designed using the Ackermann methodology. The observer is employed to estimate the unmeasurable variables of virtual patients which are generated by Bergman's model. Once the unmeasurable state variables are obtained by the Luenberger observer using only the input-output information of the Bergman's model, a control algorithm based on eigenvalues relocation trough Ackermann methodology for linear systems is applied. In this methodology, a constant feedback gain vector is obtained in order to compute the control signal (insulin) to be applied to virtual patient and keep on normoglycemia rank. The carbohydrates ingestion is considered as the main disturbances. In order to assess the proposed methodology, two tests are designed: the first one consists of changing the reference signal in order to evaluate the control sensitivity; and the second one includes different proportions of prandial insulin used in open-loop to try the controller response under distinct operation conditions. The results are obtained via simulation using Simulink of Matlab.
\end{abstract}

Keywords-Luenberger observer, Bergman model, artificial pancreas, type 1 diabetes.

\section{Introduction}

Type 1 diabetes Mellitus (T1DM) is a chronic disease, which occurs when the pancreas does not produce insulin, because beta-cells are destroyed. Hyperglycemia is a common effect of uncontrolled T1DM and over time leads to serious damage to many of the body's systems. Currently, 347 million people worldwide have diabetes, more than $80 \%$ of diabetes deaths occur in low and middle income countries. World Health Organization estimates that deaths caused by diabetes will double between 2005 and 2030 and will be the 7th leading cause of death in 2030.

At present, it is impossible to regenerate the beta cells and cure this disease. T1DM can be controlled by exogenous insulin supplying subcutaneous injections and by the glucose

${ }^{1}$ Onofre Orozco and Carlos E. Castañeda are with University of Guadalajara, University Center of los Lagos, Av. Enrique Díaz de León no. 1144 Colonia Paseos de la Montaña, Lagos de Moreno, Jalisco, México, 47460; phone: +52 (474) 742 4314, 7423678 Ext. 66586, Fax Ext. 66527,

${ }^{2,3}$ Agustín Rodríguez-Herrero, Gema García-Saéz and M. Elena Hernando are with Bioengineering and Telemedicine Group, Polytechnical University of Madrid, Spain. Biomedical Research Networking Centre in Bioengineering Biomaterials and Nanomedicine (CIBER-BBN), Madrid, Spain measurements using glucometers or continuous glucose subcutaneous sensors monitoring (CGM) [1]. This treatment is very invasive and painful. A serious complication of the therapies is the hypoglycemia which appears when the glucose level is lower than $70 \mathrm{mg} / \mathrm{dl}$. CGM has been improved in the last years, the measurements are more accurate and are made in real-time, with shorter sample periods. But they continued to have problems as duration, calibrations and delays [2].

Continuous subcutaneous insulin infusion systems (CSII) uses an insulin pump and reduces the therapy injections painful. CSII can be used to optimize the patient's insulin therapy and to improve lifestyle flexibility. The use of CSII together CGM is known as sensor-augmented pump (SAP) therapy [2].

The artificial pancreas (AP) should automatically keep the blood glucose levels to close normal levels, avoiding hypoglycaemia without human interaction. An AP is composed by a control algorithm that determines the amount of insulin needed and infusioned, a pump and CGM as primary source. Some of the closed-loop (CL) techniques applied in diabetes to design the control algorithm include PID (proportional-integral-derivative) approximations, model predictive control, fuzzy control, and robust control. Hybrid closed-loop (HCL) control has been also evaluated as a means to reduce the peak postprandial glucose levels [3].

The majority of control algorithms need a model of the plant (system to control, in T1DM the patient) to be designed, fitted or implemented. The availability of a glucose model to simulate the behavior in particular of T1DM patients gives the possibility of design and evaluate insulin infusion algorithms.

In T1DM the most common models are based in compartments. A glucose compartmental model is a set of nonlinear equations that represents the glucose and insulin masses or concentrations. The set of equations emulates the glucoregulatory system trying to describe the dynamics of blood glucose or subcutaneous glucose through the insulin and meals.

Sometimes the real experiments cannot be done at all, because they are dangerous in vivo, difficult or not ethical. There are several compartmental models to evaluate the glucose on people with T1DM. In the last ten years, two have been the compartmentals models widely used: 1) Bergman's model (BM) also known as minimal model, which consider only the glucose and insulin compartments and the interaction between them [4]; and 2) Hovorka's model (HM) in which the insulin has 3 different sensibilities on glucose distribution/transport, disposal, and endogenous production [5], [6]. Cobelli's model in which patient has daily events and 
a submodel of the digestive track is used to determinate the glucose concentration [7].

T1DM patients have a lot of problems to fit the insulin therapy to their life habits. The main problem is not to use too much insulin in order to avoid hypoglycemia events. The goal of all alternative treatments in T1DM is to keep the glucose concentration in normal ranges using as much doses of exogenous insulin as necessary. CGM and CSII require the subcutaneous route, which complicates control because of delays in both routes, measurement and insulin action.

In control design, it is difficult to infer the behavior of the nonlinear systems, which have internal or external disturbances, unknown parameters or unmodeled dynamics and delays. When a control law it is designed using a mathematical model, is necessary to estimate the unmeasurable variables. A useful tool to estimate the unmeasurable variables are the observers; there exists a wide variety of them applied in very different fields successfully as Kalman filter, observers with sliding modes, nonlinear observers and Luenberger observer [8-9].

In this work we propose to design a Luenberger observer in order to estimate the unmeasurable state space variables (as it is done in [10]). The HM is linearized and an operation point is selected to evaluate the model; this linearized model is used to design the Luenberger observer. The observer based on the HM is employed to estimate the unmeasurable variables of virtual patients (VP) generated by BM. Once the unmeasurable state variables are obtained by the Luenberger observer using only the input-output information (insulinglucose) of the BM, a control algorithm based on eigenvalues relocation trough Ackermann methodology for linear systems is applied. In this methodology, a constant feedback gain vector is obtained in order to compute the control signal (insulin) to be applied to VP and keep on normoglycemic rank. The main external disturbances included in this work is the carbohydrates (CHOs) ingestion. Two tests are designed to evaluated the performance of the control algorithm: 1) Target sensibility, it consists of changing the reference signal in order to evaluate the steady state control; and 2) Prandial sensibility, it includes different proportions of prandial insulin used in open-loop (OL) or CSII to try the controller response under distinct operation conditions.

\section{Hovorka's model}

HM is one of the most accepted models describing the glucose-insulin dynamic. Roman Hovorka modelled the diabetes disease with eight compartmental differential equations in 2004 [6] and in subsequent works updated his model including the equation of CGM sensor [11]. HM is represented in states space as:

$$
\begin{aligned}
& \dot{C}=k_{a}[G(t)-C] \\
& \dot{Q}_{1}=-\left[\frac{f_{01}^{c}}{V_{G} G(t)}-X_{1}\right] Q_{1}+k_{12} Q_{2}-f_{R}+E G P_{0}\left[1-X_{3}\right]+U_{G}(t)
\end{aligned}
$$

$$
\begin{aligned}
& \dot{Q}_{2}=X_{1} Q_{1}-\left[k_{12}+X_{2}\right] Q_{2} \\
& \dot{X}_{1}=-k_{a 1} X_{1}+k_{b 1} I \\
& \dot{X}_{2}=-k_{a 2} X_{2}+k_{b 2} I \\
& \dot{X}_{3}=-k_{a 3} X_{3}+k_{b 3} I \\
& \dot{I}=\frac{S_{2}}{V_{I} t_{\max , I}}-k_{e} I \\
& \dot{S}_{2}=\frac{S_{1}}{t_{\max , I}}-\frac{S_{2}}{t_{\max , I}} \\
& \dot{S}_{1}=u(t)-\frac{S_{1}}{t_{\max , I}}
\end{aligned}
$$

$S_{2}$ and $S_{1}$ are two-compartments chain representing absorption of subcutaneously administered short-acting insulin $(\mathrm{mU})$; $G(t)=Q_{1} / V_{G}$ is the glucose concentration in the plasma $(\mathrm{mg} / \mathrm{dl})$; $V_{G}$ is the blood volume $(l / k g) ; u(t)$ is input signal, represents the infusion dose of insulin $(U)$; and $E G P_{0}$ represents the endogenous glucose production liver extrapolated to a 0 insulin concentration $(\mathrm{mg} / \mathrm{dl})$.

The Glucose absorption is a fundamental process affecting postprandial glucose excursions. In HM (1), the gut absorption rate $U_{G}(t)(\mathrm{mg} / \mathrm{min})$ is represented by:

$$
U_{G}(t)=\frac{d(t) A_{G} t e^{\frac{-t}{t_{\max , G}}}}{{ }_{\max , G}},
$$

where $t_{\max , G}$ is the time-of-maximum appearance rate of glucose in the accessible glucose compartment, $d(t)$ is the amount $(m g)$ of CHOs ingested, $A_{G}$ is carbohydrate bioavailability.

The renal glucose clearance $\left(f_{R}\right)$ appears when the glucose is above threshold of $9 \mathrm{mmol} / \mathrm{l}$, which can be represented as:

$$
f_{R}=\left\{\begin{array}{cc}
0.003(G(t)-9) V_{G} & \text { if } G(t) \geq 9 \mathrm{mmol} \mathrm{L}^{-1} \\
0 & \text { otherwise }
\end{array},\right.
$$

The total non-insulin-dependent glucose flux $\left(f_{0 I}{ }^{\mathrm{c}}\right)$ corrected depends on glucose concentration, it is represented as:

$$
f_{01}^{c}=\left\{\begin{array}{cc}
f_{01} & \text { if } G(t) \geq 4.5 \mathrm{mmol} L^{-1} \\
f_{01} \frac{G(t)}{4.5} & \text { otherwise }
\end{array} .\right.
$$

In this work, the HM (1) is linearized around of an operation point in order to design the Luenberger observer. This observer is used to design a control law by eigenvalues relocation. This procedure is explained in section III. The HM (1) and its parameters are obtained from [5-6] and are used to parameterize the observer during simulations.

\section{Proposed methodology}

In this section, we proposed the procedure for designing the Luenberger observer in the T1DM HM and applying it in $\mathrm{BM}$ to $\mathrm{CL}$. 


\section{A. Luenberger observer}

The assumption that all state variables are available for feedback may not hold in practice, because in the case of T1DM nowadays it is not feasible to measure all HM variables for an ambulatory AP. In this paper, HM (1) can be represented in a general form as:

$$
\begin{aligned}
& \dot{\boldsymbol{x}}=\boldsymbol{f}(\boldsymbol{x})+\boldsymbol{g}(\boldsymbol{x}) u+\boldsymbol{d}(t), \\
& y=h(\boldsymbol{x}),
\end{aligned}
$$

where $\boldsymbol{f}(\boldsymbol{x})$ and $\boldsymbol{g}(\boldsymbol{x})$ are nonlinear functions; $\boldsymbol{d}(t)$ represents the external disturbances (described in equation (2)) and $h(x)$ is the output function.

In [10], the Luenberger observer linear theory is extended for general nonlinear system (5) with following structure:

$$
\dot{\hat{\boldsymbol{x}}}=\boldsymbol{f}(\hat{\boldsymbol{x}})+\boldsymbol{g}(\hat{\boldsymbol{x}}) u+\boldsymbol{d}(t)+\boldsymbol{l}(y-h(\hat{\boldsymbol{x}})),
$$

where the Luenberger gain vector $l$ is calculated by eigenvalues relocation trough Ackermann methodology, $\hat{\boldsymbol{x}}$ is the estimated states vector for $\boldsymbol{x}$. In order to select the desired eigenvalues, the system (5) is linearized using the Jacobian to obtain:

$$
\mathbf{A}=\left.\frac{\partial \boldsymbol{f}}{\partial \boldsymbol{x}}\right|_{x=\rho}, \mathbf{b}=\left.\frac{\partial \boldsymbol{g}}{\partial u}\right|_{x=\rho}, \mathbf{c}=\left.\frac{\partial h}{\partial \boldsymbol{x}}\right|_{x=\rho}
$$

where $\mathbf{A}$ is the state matrix and $\mathbf{b}$ is the input vector and $\mathbf{c}$ is the output vector. The operation point $\rho$ is selected when $y=90$ $\mathrm{mg} / \mathrm{dl}$. That is, the T1DM patient is into normoglycemia band. Then, the linearized system is:

$$
\begin{aligned}
& \dot{\boldsymbol{x}}=\mathbf{A} \boldsymbol{x}+\mathbf{b} u+\boldsymbol{d}(t), \\
& y=\mathbf{c x} .
\end{aligned}
$$

The pair (A,c) from (8) is completely state observable because

$$
\operatorname{rank}(\mathcal{O})=\operatorname{rank}\left(\left[\begin{array}{llll}
\mathbf{c} & \mathbf{c A} & \cdots & \mathbf{c} \mathbf{A}^{n-1}
\end{array}\right]^{\top}\right)=n,
$$

where $\mathcal{O}$ is the observability matrix. The desired eigenvalues are relocated to the left of eigenvalues of $\mathbf{A}$ in the left side of the complex " $s$ " plane. This is done by computing the gain vector $\boldsymbol{l}$ with Ackermann methodology as follows [12]:

$$
\boldsymbol{l}=\phi(\mathbf{A}) \mathcal{O}^{-1}\left[\begin{array}{llll}
0 & \cdots & 0 & 1
\end{array}\right]^{\top},
$$

where $\phi(\mathbf{A})$ results from desired characteristic polynomial for (8). The observer (6) will estimate the unmeasurable state variables of HM (1) with the form (5). Even when output of system (1) is considered available for measuring, in this work the output estimation of $y=C=x_{1}$ in system (1) will contribute to estimate the state variable $G$ in BM.

\section{B. Control algorithm}

With all the state space variables $\boldsymbol{x}$ available for feedback, it is possible to propose a control law using the gain feedback concept. In order to obtain the feedback gains $\boldsymbol{k}$ to calculate the control signal $u_{c}(t)$, we use the Ackermann methodology. This is possible by applying the duality theorem for controllability-observability [13]. As the pair $(\mathbf{A}, \mathbf{b})$ of system (8) is completely state controllable due to controllability matrix

$$
\mathcal{C}=\left[\begin{array}{llll}
\mathbf{b} & \mathbf{A b} & \cdots & \mathbf{A}^{n-1} \mathbf{b}
\end{array}\right],
$$

has full rank. The feedback control gains are computed with Ackermann method as:

$$
\boldsymbol{k}=\left[\begin{array}{llll}
0 & \cdots & 0 & 1
\end{array}\right] \mathcal{C}^{-1} \varphi(\mathbf{A})
$$

where $\varphi(\mathbf{A})$ results from the desired control characteristic polynomial for (8). The control eigenvalues are relocated at the left of their corresponding with $\mathbf{A}$ at the right of the observer eigenvalues, in the left side of the complex " $s$ " plane. The control linear theory is applied to feedback estimated states of nonlinear system that is the BM, which is described in section IV. In this work, the HM is parameterized with the mean of six patients shown in [5-6].

\section{Iv. Test methodology}

In this work, it is proposed to test the observer and controller with a VP different from HM. Figure 1 depicts the employed configuration to test the designed controller using the estimated variables by the Luenberger observer.

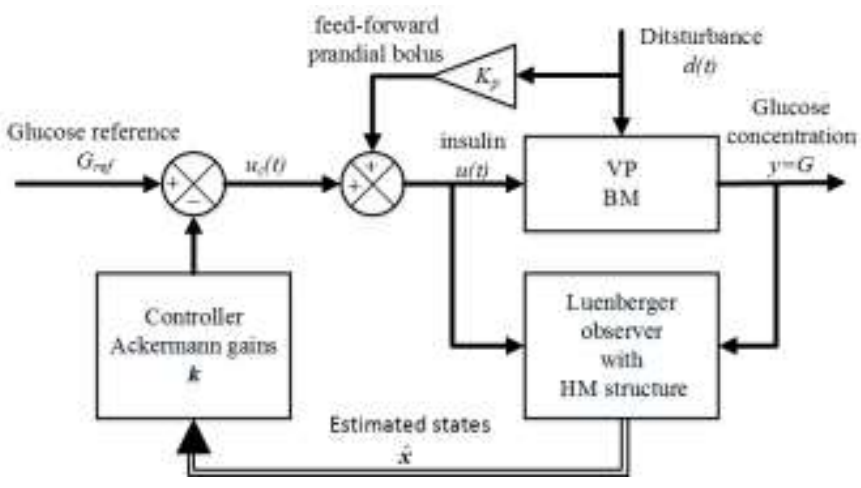

Figure 1. Configuration structure with controller coupled to Luenberger observer with test VP.

Both controller and observer are based on HM. BM is used as VP test taking only BM output and input to estimate the unmeasurable states. All the estimated variables are used in the controller. $K_{p}$ is adjusted during the different experiments which are described in subsection IV-B.

\section{A. Virtual patient}

$\mathrm{BM}$ also known as minimal model is used in this work to generate the VP. Insulin absorption equations are added to 
original BM [14] to consider the insulin delays having the next state space representation:

$$
\begin{aligned}
& \dot{G}=-p_{1}\left[G-G_{b}\right]-X G+d(t) \\
& \dot{X}=-p_{2} X+p_{3}\left[I-I_{b}\right] \\
& \dot{I}=-\eta\left[I-I_{b}\right]+\gamma[G-h]^{+} t+\frac{S_{2}}{V_{I} t_{\max , I}} \\
& \dot{S}_{2}=\frac{S_{1}}{t_{\max , I}}-\frac{S_{2}}{t_{\max , I}} \\
& \dot{S}_{1}=u(t)-\frac{S_{1}}{t_{\max , I}}
\end{aligned}
$$

where $G$ is the plasma glucose $(m g / d l), X$ is the insulin effect over glucose elimination $(1 / \mathrm{min}), I$ is the plasma insulin $(m U / d l) S 1$ and $S 2$ are the two compartments where the insulin is administered in subcutaneous route $(m U)$. The parameters to generate the VP population with BM are taken from [4].

\section{B. Pump simulation description}

The operation of an insulin pump is simulated as follows: 1) as the simulation iterates at 1 second and the pump infusion a bolus each 5 minutes, the control signal uc(t) is accumulated during this 5 minutes; 2) as insulin pumps cannot infusion any exact amount of insulin due to its minimum dosage ability, the insulin to be administered to the patient by the pump is adjusted to pump resolution dosage, the rounding error is added for the next infusion time; 3) if the control signal is negative (the controller indicates to extract insulin, which is impossible in real life), the insulin pump action is suspended and counts the suspension time; for each 30 minutes of suspension the pump dose a microbolus ergo minimum dosage resolution in order to avoid the catheter occlusion by insulin crystallization.

\section{Implementation scenarios}

To stimulate the glucose-insulin dynamic, the VP are disturbed with a diet of CHOs as follows: 40 gr at 7:30 hrs. during breakfast; $15 \mathrm{gr}$ at 11:00 hrs. in a first snack; $90 \mathrm{gr}$ for lunch at 13:00 hrs.; $80 \mathrm{gr}$ for dinner at 19:00 horas and $15 \mathrm{gr}$ for a second snack at 23:00 hrs. [15].

Three different experiments are proposed to see the performance of the controller under different conditions.

\section{1) Scenario 1: Target sensibility}

In this experiment the reference signal is changed in order to evaluate the steady state control. The percentage of the feed-forward prandial bolus insulin $K_{p}$ is set to $50 \%$. The sensitivity to reference signal is tested using four different values $(80,90,100$ and $110 \mathrm{mg} / \mathrm{dl})$ for each VP.

\section{2) Scenario 2: Prandial sensibility}

Different proportions of prandial insulin used in CSII is administrated independently of controller. The reference is placed in $90 \mathrm{mg} / \mathrm{dl}$. In order to test the control algorithm under distinct operation conditions, the feed-forward proportions of prandial bolus insulin $K_{p}$ is adjusted in $0 \%, 50 \%, 60 \%$ and $80 \%$.

\section{v. Results and discussion}

The observer is used to estimate the unmeasurable variables of the BM. In Figure $2 \mathrm{~A}$ ) is shown the estimation of the HM output $y_{H}=C$ and its corresponding identification error $e_{H}=y_{H}-\hat{y}_{H}$ is shown in Figure $2 \mathrm{~B}$ ). As can be seen in these Figures, the variable $C$ is estimated with a FIT $=99 \%$, it is important to mention that the results shown in [10] present the estimation of all variables. In this simulation results, 24 hours are presented based on the results obtained in the estimation of HM variables, the proposed algorithm is used in $\mathrm{BM}$. The estimation error is bounded in $0<e_{H}<1.5 \mathrm{mg} / \mathrm{dl}$.

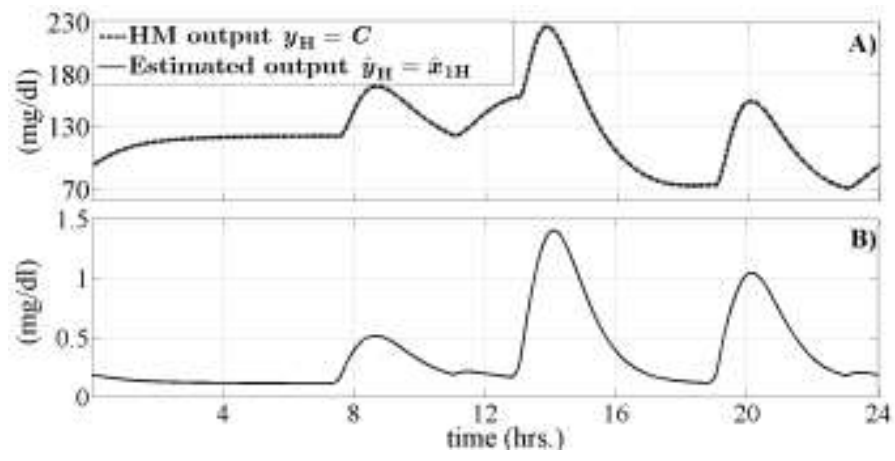

Figure 2. A) Estimation of HM output $y_{H}=C$ (dashed gray line), and its estimated output $\hat{y}_{H}=\hat{x}_{1 \mathrm{H}}$ (continuous black line); and B) estimation error

$$
e_{H}=y_{H}-\hat{y}_{H} \text {. }
$$

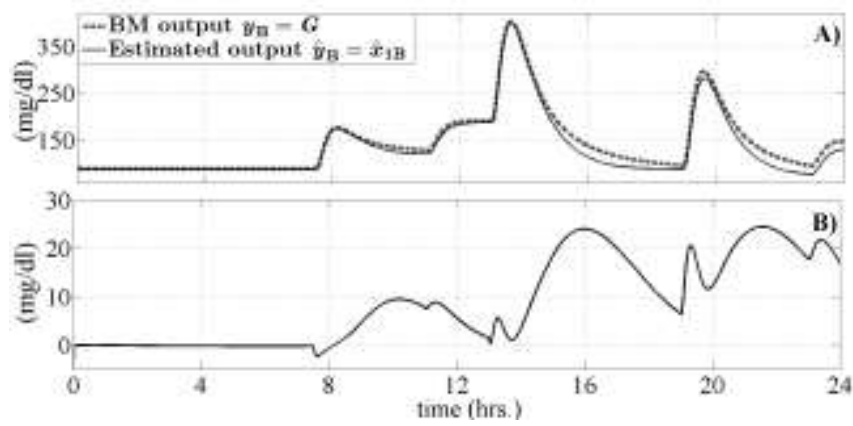

Figure 3. A) Estimation of BM output $y_{B}=G$ (dashed gray line), and its estimated output $\hat{y}_{B}=\hat{x}_{1 \mathrm{~B}}$ (continuous black line); and B) estimation error

$$
e_{B}=y_{B}-\hat{y}_{B} .
$$

Figures 3A) and 3B) depict the estimation of BM output $y_{B}=G$ and its corresponding estimation error $e_{B}=y_{B}-\hat{y}_{B}$, respectively. The output variable $y_{B}=G$ is estimated with a FIT $=98 \%$, the error is contained between $-2<e_{B}<25 \mathrm{mg} / \mathrm{dl}$. This error is due to whole differences between models. The goal for estimation the BM output consists of using this 
estimated variable in order to control it applying the procedure explained in section III B) using the two different scenarios.

\section{1) Scenario 1: Target sensibility}

In Figure $4 \mathrm{~A}$ ) is displayed the $\mathrm{BM}$ controlled output $y_{B}=G$ of VP\#2 comparing the OL with CL therapy to a reference $G_{\text {ref }}=90 \mathrm{mg} / \mathrm{dl}$; Figure $4 \mathrm{~B}$ ) shown the insulin administered $u(t)$ to VP\#2, which includes $K_{p}=50 \%$ of $\mathrm{OL}$ prandial bolus $u_{O L}(t)$ added to $u_{c}(t)$ computed by the controller with Ackermann methodology described in section III B) and shown in Figure 1. From Figure 4 A) is seen that the CL glucose, using the estimated variables, is $1.4 \%$ of the day in hyperglycemia than $4.1 \%$ of OL glucose. Both OL and CL glucose avoids hypoglycemia level this means hypoglycemia time is $0 \%$; but, as controller is limited to sidedness, is not possible to increase the glucose level until other disturbance appears.

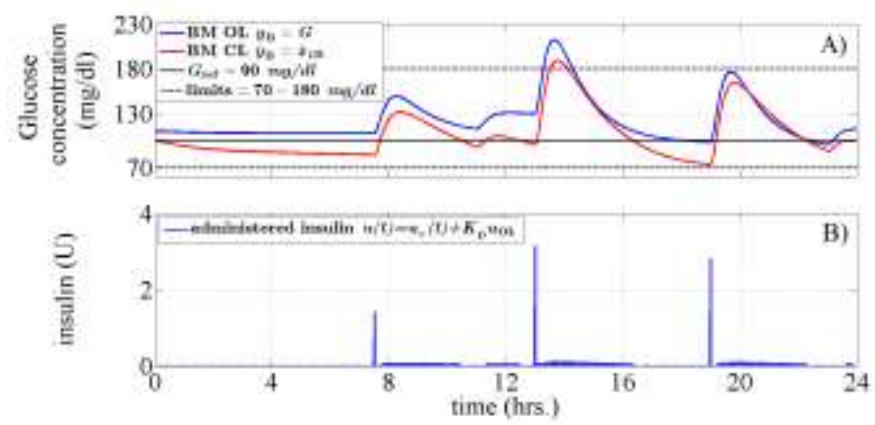

Figure 4. Particular case of control applied to VP\#2 of BM A) BM OL output $y_{B}=G$ is compared compared with CL output $\hat{y}_{B}=\hat{x}_{1 B}$; in B) shows the administered insulin $u(t)=u_{c}(t)+K_{p} u_{O L}$.

Figure 5 and Figure 6 display the mean OL output $y_{B}=G$ with maximum and minimum VP values (blue areas) versus mean CL output $\hat{y}_{B}=\hat{x}_{1 B}$ with maximum and minimum VP values (red areas); in CL the control signal $u(t)=u_{c}(t)+K_{p} u_{O L}$ with $K_{\mathrm{p}}=50 \%$.

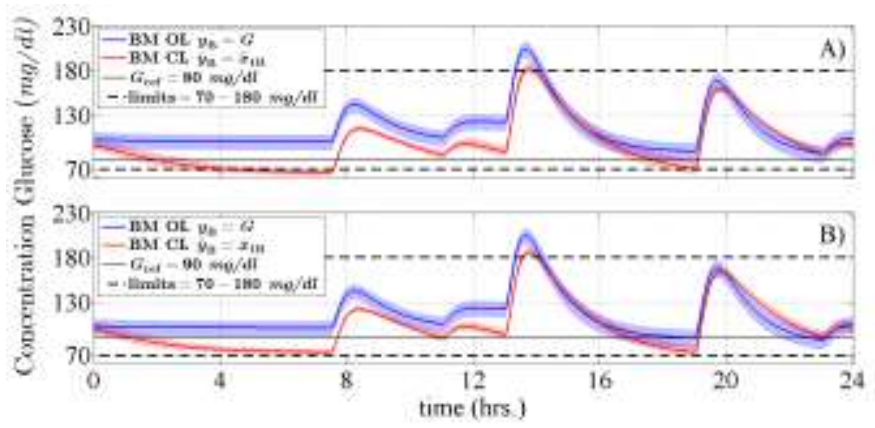

Figure 5. VP population of BM OL output $y_{B}=G$ is compared compared with VP population in CL output $\hat{y}_{B}=\hat{x}_{1 B}$, prandial bolus feed-forward $K_{p}=50 \%$ A) $G_{r e f}=80 \mathrm{mg} / \mathrm{dl}$; B) $G_{r e f}=90 \mathrm{mg} / \mathrm{dl}$.
Figure 5 compares OL and CL outputs, for all OL the mean time in hyperglycemia is $4.1 \%$ and $0 \%$ in hypoglycemia. In Figure $5 \mathrm{~A}) G_{\text {ref }}=80 \mathrm{mg} / \mathrm{dl}$ the $\mathrm{CL}$ mean time in hyperglycemia is $0.9 \%$ and in hypoglycemia is $14.1 \%$. For Figure 5 B) $G_{\text {ref }}=90 \mathrm{mg} / \mathrm{dl}$, the $\mathrm{CL}$ mean time in hyperglycemia is $1.7 \%$ and in hypoglycemia is $0.3 \%$.

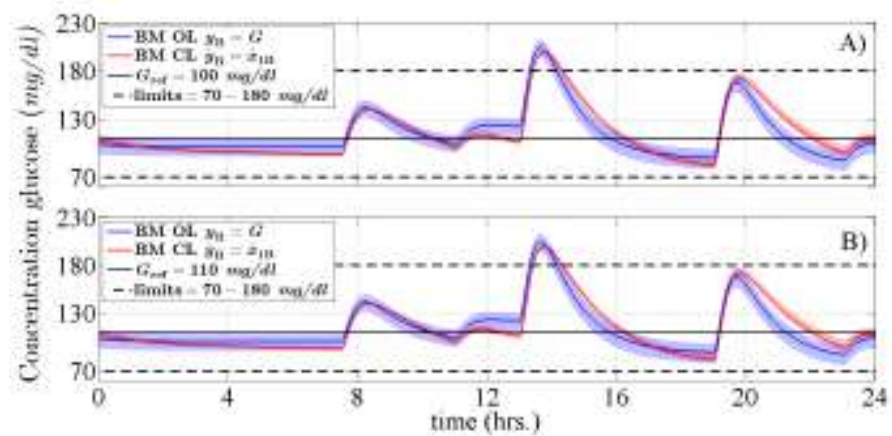

Figure 6. VP population of BM OL output $y_{B}=G$ is compared compared with VP population in CL output $\hat{y}_{B}=\hat{x}_{1 B}$, prandial bolus feed-forward

$$
K_{p}=50 \% \text { A) } G_{r e f}=100 \mathrm{mg} / \mathrm{dl} \text {; B) } G_{r e f}=110 \mathrm{mg} / \mathrm{dl} \text {. }
$$

Comparisons between OL and CL outputs in Figure $6 \mathrm{~A}$ ) $G_{\text {ref }}=100 \mathrm{mg} / \mathrm{dl}$ the CL mean time in hyperglycemia is $2.6 \%$ and in hypoglycemia is $0 \%$. $6 \mathrm{~B}) G_{\text {ref }}=110 \mathrm{mg} / \mathrm{dl}$, the $\mathrm{CL}$ mean time in hyperglycemia is $3.7 \%$ and in hypoglycemia is $0 \%$. From the times in hypoglycemia level is possible deduce that if $G_{\text {ref }}$ is raised the risk of reach hypoglycemia is reduced; then raising the reference hypoglycemia risk is avoided but, in consecuense is few increased the hyperglycemia period.

\section{2) Scenario 2: Prandial sensibility}

Figure 7 and Figure 8 depict the mean OL output $y_{B}=G$ with maximum and minimum VP values (blue areas) versus mean CL output $\hat{y}_{B}=\hat{x}_{1 B}$ with maximum and minimum VP values (red areas); in CL the reference signal $G_{\text {ref }}=90 \mathrm{mg} / \mathrm{dl}$ for the four trials.

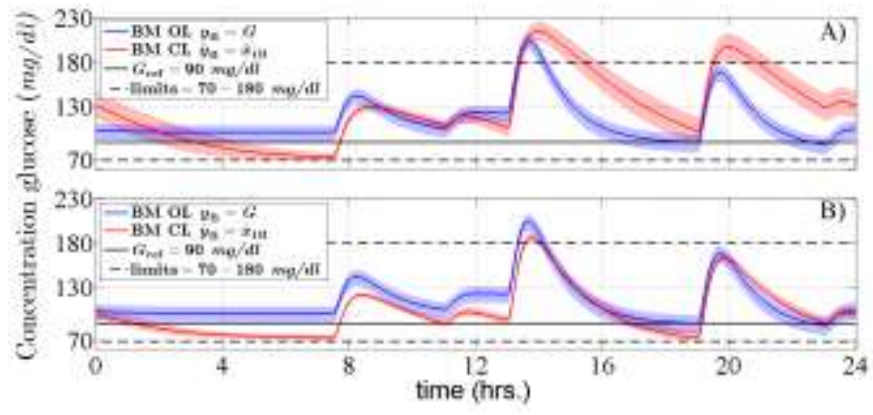

Figure 7. Comparation between VP population of BM in OL output $y_{B}=G$ versus CL output $\hat{y}_{B}=\hat{x}_{1 B}$, with $G_{r e f}=90 \mathrm{mg} / \mathrm{dl}$; A) prandial bolus feedforward $K_{p}=0 \%$ and B) prandial bolus feed-forward $K_{p}=50 \%$. 
Figure 7 shows OL and CL outputs, in A) control signal is adjusted with $K_{\mathrm{p}}=0 \%$, computing the CL mean time in hyperglycemia is $13.6 \%$ and in hypoglycemia is $2.0 \%$; in B) control signal is complemented with $K_{\mathrm{p}}=50 \%$, the calculus of CL mean time in hyperglycemia indicates $1.7 \%$ and for hypoglycemia is $0.3 \%$.

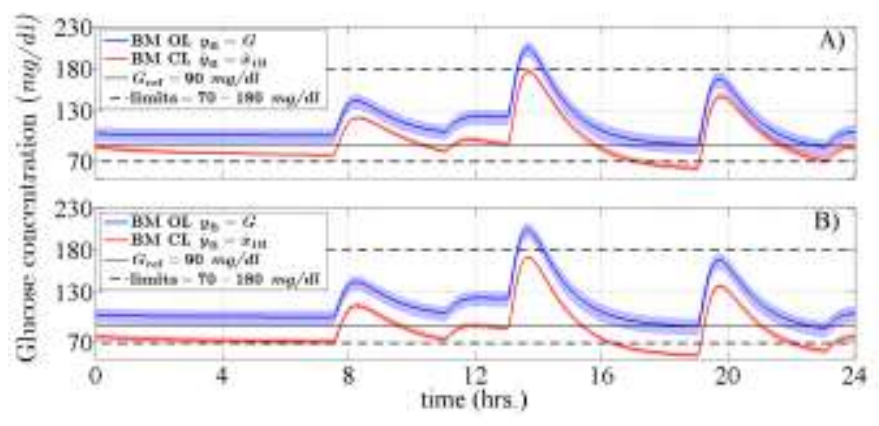

Figure 8. Comparation of BM VP population between OL output $y_{B}=G$ and CL output $\hat{y}_{B}=\hat{x}_{1 B}$, with $\left.G_{r e f}=90 \mathrm{mg} / \mathrm{dl} ; \mathrm{A}\right)$ prandial bolus feedforward $K_{p}=80 \%$ and B) prandial bolus feed-forward $K_{p}=100 \%$.

With reference signal fixed in $G_{r e f}=90 \mathrm{mg} / \mathrm{dl}$, in Figure 8 A) the control signal is balanced with $K_{\mathrm{p}}=80 \%$, the calculation of CL mean time in hyperglycemia results $0 \%$ and for hypoglycemia $8.2 \%$. In Figure $8 \mathrm{~B}$ ) the control signal is made-up with $K_{\mathrm{p}}=100 \%$, the percentage of CL mean time in hyperglycemia indicates $0 \%$ and for hypoglycemia is $23.6 \%$. From trials in Figure 7 and Figure 8 is deduced that, in full CL i.e. $K_{\mathrm{p}}=0 \%$ the controller is able to deal with the disturbances and regulates the glucose output, taking it to normoglycemic range. According proportion of prandial bolus feed-forward $K_{\mathrm{p}}$ is increasing the output regulation is more time in the normoglycemic band. But, if $K_{\mathrm{p}}=100 \%$ i.e. whole prandial bolus is administered the VP is hyper insulinized, becase in OL the VP is regulated and more insulin is added to its therapy.

\section{vI. Conclusion}

From results we conclude that proposed controller is able to deal with disturbances to regulate glucose concentration in VP with T1DM. The control operation conditions must be selected carefully in order to avoid hypoglycemia events and reducing the time in hyperglycemic level. The control algorithm based in Luenberger observer which estimates the unmeasurable variables absorbing the intra patient variability and external disturbances its good strategy to tackle the AP challenge.

\section{Acknowledgment}

Authors thanks to CONACYT (México) under scholarship number 284966 and to retention program 120489.

\section{References}

[1] DCCT Research Group 1993, "The effect of intensive treatment of diabetes on the development and progression of long-term complications in insulin-dependent diabetes mellitus", New England Journal of Medicine, vol. 329, pp. 977-986

[2] Forlenza, G. P., Buckingham, B., \& Maahs, D. M., "Progress in diabetes technology: developments in insulin pumps, continuous glucose monitors, and progress towards the artificial pancreas". J Pediatr, 169, 13-20, 2016.

[3] Agustín Rodríguez-Herrero, Carmen Pérez-Gandía, Mercedes Rigla, Alberto de Leiva, Enrique J. Gómez and M. Elena Hernando, "A Simulation Study of an Inverse Controller for Closed- and SemiclosedLoop Control in Type 1 Diabetes", Diabetes Technology \& Therapeutics, Volume 12, Number 2, 2010.

[4] Kaveh, P., and Shtessel, Y. B., "Blood glucose regulation via double loop higher order sliding mode control and multiple sampling rate". In Modern Sliding Mode Control Theory. Springer Berlin Heidelberg, pp. 427-445, 2008.

[5] Hovorka Roman, Valentina Canonico, Ludovic J Chassin, Urich Haueter, Massimo Massi-Benedetti, Marco Orsini Federici, Thomas R Pieber, Helga C Schaller, Lukas Schaupp, Thomas Vering, and Malgorzata E Wilinska, "Nonlinear model predictive control of glucose concentration in subjects with type 1 diabetes", Institute of Physics Publishing Physiological Measurement, Am J Physiol Endocrinol Metab 282: E992E1007, 2002.

[6] Hovorka Roman, Fariba Shojaee-Moradie, Paul V. Carroll, Ludovic J. Chassin, Ian J. Gowrie, Nicola C. Jackson, Romulus S. Tudor, A. Margot Umpleby, and Richard H. Jones, "Partitioning glucose distribution/transport, disposal, and endogenous production during IVGTT", The American Physiological Society, Physiol. Meas. 25, 2004, 9059200 .

[7] Chiara Dalla Man, Robert A. Rizza, and Claudio Cobelli, "Meal Simulation Model of the Glucose-Insulin System", IEEE Transactions on Biomedical Engineering, Vol. 54, No. 10, october 2007.

[8] D., Boiroux, Duun-Henriksen, A. K., Schmidt, S., Nørgaard, K., Poulsen, N. K., Madsen, H., and Jørgensen, J. B. "Adaptive control in an artificial pancreas for people with type 1 diabetes". Control Engineering Practice, 58, 332-342, 2017.

[9] Gonzalez, A. A., Voos, H., and Darouach, M. "States and unknown input estimation via non-linear sliding mode high-gain observers for a glucose-insulin system". In Biomedical Engineering and Sciences (IECBES), 2016 IEEE EMBS Conference on (pp. 388-393). IEEE, 2016, December.

[10] Orozco, O., Castañeda Hernández, C. E., Rodríguez Herrero, A., García Saéz, G., Pérez, H., and Elena, M. "Luenberger observer with nonlinear structure applied to diabetes type 1". $8^{\text {th }}$ International Supercomputing Conference in México, ISUM 2017.

[11] Wilinska, M. E., Chassin, L. J., Acerini, C. L., Allen, J. M., Dunger, D. B., and Hovorka, R. "Simulation environment to evaluate closed-loop insulin delivery systems in type 1 diabetes". Journal of diabetes science and technology, 4(1), 132-144, 2010.

[12] Ogata, K., Modern control engineering, 5th edition. Upper Saddle River, New Jersey, 07458, 2009.

[13] Chen, C. T., Linear system theory and design. Oxford University Press, Inc., 1995.

[14] Boiroux, D., Bátora, V., Hagdrup, M., Tárnik, M., Murgaš, J., Schmidt, S., ... and Jørgensen, J. B., "Comparison of Prediction Models for a Dual-Hormone Artificial Pancreas". Funded by the Danish Diabetes Academy supported by the Novo Nordisk Foundation. Contact information: John Bagterp J_rgensen (jbjo@ dtu. dk). IFACPapersOnLine, 48(20), 7-12, 2015.

[15] Anguita, C., Caixás, A., Clapés, J., Claveria, M., Gallach, I., and Jansá, M., "Documento de Consenso sobre Recomendaciones Nutricionales y de Educación Alimentaria en la Diabetes". Barcelona: Associació Catalana de Diabetes, 2003. 